\title{
Concurrencia de turistas en el Paseo de la Juventud y plan de dinamización para su inserción en la oferta dentro del circuito turístico de Guayaquil, Ecuador
}

\section{Tourist participation on the Paseo de la Juventud and revitalization plan for its inclusion on the tourist circuit in Guayaquil, Ecuador}

Elsie Zerda B. ${ }^{1}$, Aline Gutiérrez N ${ }^{2}$, María Auxiliadora Cruz S. ${ }^{3}$, Gabriela Cali N ${ }^{3}$

Resumen: Guayaquil es la ciudad con mayor densidad poblacional del Ecuador. En los últimos años se han realizado importantes regeneraciones urbanas, especialmente en el centro de la ciudad el que se ha convertido en un sitio clave para el desarrollo de actividades tanto de turistas como de residentes. La campaña de promoción turística “Guayaquil es mi Destino”, impulsada por el Municipio de la ciudad de Guayaquil, incluye entre sus atractivos turísticos el Paseo de la Juventud, conocido informalmente como Puente Zigzag; esta campaña no ha generado el interés esperado. El objetivo de este trabajo fue analizar cómo influye la concurrencia de turistas en el Paseo de la Juventud mediante un estudio de campo para proponer un plan de dinamización que permita su inserción en la oferta dentro del circuito turístico de Guayaquil. El plan contempla que el Paseo de la Juventud se convierta en un punto de información turística de la ciudad Guayaquil, además de un lugar de realización de eventos culturales para jóvenes y de difusión de la cultura a través de la venta de artesanías. Esto implica que el plan de dinamización tiene dos ejes principales, la implementación o mejoras desde el equipamiento, iluminación y seguridad hasta un aspecto que se enfoque al ámbito ambiental, además de la inclusión del Paseo de la Juventud en el circuito turístico de la ciudad de Guayaquil.

Palabras clave: Atractivos turísticos, plan de dinamización, regeneración urbana, circuito turístico.
Abstract: Guayaquil is the most densely populated city in Ecuador. In recent years, significant urban regenerations have been undertaken, particularly in the city center, which has become a key site for activities enjoyed by tourists and residents alike. The tourism promotion campaign, "Guayaquil is my Destination", driven by the Municipality of Guayaquil, includes among its tourist attractions the Paseo de la Juventud, informally knows as the Zigzag Bridge. This campaign has not aroused the interest expected. The aim of this study was to analyze the influence of the convergence of tourists on the Paseo de la Juventud using a field study to propose a revitalization plan that makes possible its inclusion on Guayaquil's tourist circuit. The plan covers the Paseo de la Juventud becoming a tourist information point in Guayaquil, as well as a place for cultural events for young people and for the dissemination of the culture through the sale of handicrafts. This implies that the revitalization plan has two main axes: the implementation or improvements made from equipment, illumination and safety to an aspect that focuses on the environment, and the inclusion of the Paseo de la Juventud on the tourist circuit of Guayaquil.

Key words: Tourist attractions, revitalization plan, urban regeneration, tourist circuit.

\section{(Presentado: 25 de enero de 2018. Aceptado: 25 de septiembre de 2018)}

\footnotetext{
Magíster en Administración de Empresas. Universidad Católica de Santiago de Guayaquil, Facultad de Especialidades Empresariales, Guayaquil, Ecuador. E-mail: elsie.zerdaacu.ucsg.edu.ec

2 Magíster en Gestión de Proyectos. Universidad Católica de Santiago de Guayaquil, Ecuador. E-mail: aline.gutierrezącu.ucsg.edu.ec

3 Ingeniero en Administración de Empresas Turísticas y Hoteleras. Universidad Católica de Santiago de Guayaquil, Ecuador. E-mail: mariuxcahotmail.com ; gabriela.cali93@gmail.com
} 


\section{INTRODUCCIÓN}

Guayaquil es la ciudad del Ecuador con mayor densidad poblacional con cerca de 2.7 millones de habitantes. La administración de la ciudad ha realizado varias regeneraciones urbanas en sus fachadas y en sus recursos turísticos en los últimos años que representan cambios importantes para su población. El centro de la ciudad es uno de los lugares donde esta regeneración ha sido más frecuente, convirtiéndose en sitio clave para el desarrollo de actividades tanto de turistas como de residentes. El centro de la ciudad de Guayaquil es un lugar fundamental para los turistas nacionales y extranjeros, ya que es la parte más antigua. Lugares como el Malecón 2000, Barrio Las Peñas y Malecón del Salado son algunas áreas donde la regeneración urbana se ha hecho presente para activar el turismo en la ciudad.

Una de las obras inauguradas en el año 2012 fue el denominado Paseo de la Juventud, conocido informalmente como Puente Zigzag debido a su construcción; su nombre se debe a que está ubicado sobre el Estero Salado, el cual une el parque lineal que queda frente a la Universidad Católica de Santiago de Guayaquil con el Malecón de la Universidad de Guayaquil y la Av. Kennedy a la altura del puente Urdesa, que congrega uno de los barrios más tradicionales de la ciudad. El Paseo de la Juventud es un paso peatonal de 220 metros de largo con seis metros de ancho. Los atractivos que nos brinda este puente es su entorno natural, ya que bordea el estero, su manglar y su arquitectónico modelo en forma de zigzag. Esta obra tiene el potencial para incorporarse a la oferta turística de Guayaquil; sin embargo, actualmente no es conocida por turistas nacionales o extranjeros, puesto que no existe un atractivo turístico ancla que sea un incentivo para la visita. La campaña de promoción turística "Guayaquil es mi Destino", impulsada por el Municipio de la ciudad de Guayaquil, incluye entre sus atractivos turísticos al Paseo de la Juventud. Sin embargo, este espacio no ha generado el interés esperado. El presente artículo enfatiza la necesidad de un plan de dinamización que consolide los atractivos naturales propios del Estero Salado y su manglar con el atractivo cultural generado a partir de la obra de regeneración urbana que conecta dos universidades tradicionales e importantes de la ciudad de Guayaquil.
El Paseo de la Juventud fue denominado así con el fin de fomentar el arte y la cultura a los jóvenes guayaquileños y el público en general. Jaramillo (2014) menciona que "es importante que se le otorgue a la ciudad la relevancia que potencialmente tiene como una urbe turística con historia y riqueza patrimonial para promocionar la ciudad a nivel internacional”. La obra, creada bajo la Fundación Siglo XXI a cargo de la Municipalidad de Guayaquil, tiene como fin principal la conexión de dos importantes avenidas del norte de la ciudad; Av. Carlos Julio Arosemena y la Av. Víctor Emilio Estrada sin un propósito turístico, facilitando el tránsito peatonal de los estudiantes de la Universidad Católica Santiago de Guayaquil y la Universidad Estatal de Guayaquil. Por este mismo motivo no se le ha dado importancia turística, debido a que se ha considerado más como un paso de tránsito de estudiantes de las universidades cercanas.

El Paseo de la Juventud (Figura 1) es un paseo peatonal que cuenta con 220 metros de longitud con una extensión de seis metros de ancho y soporta hasta ocho toneladas de peso. Tiene un diseño llamativo en forma de zigzag con seis zonas curvas a los lados externos del puente, y posee 20 metros de rampas de acceso al puente. Los materiales usados para la construcción del puente fueron adoquín y hormigón estampado. Es totalmente abierto, adaptado al entorno del lugar con ocho glorietas de jardines verticales, y áreas de observación. Está equipado con 32 sillas metálicas y de madera; así como también seis techos metálicos en formas curvilíneas ubicados a lo largo del puente. El puente tiene barandales metálicos desde el inicio hasta el fin. Cuenta con tres tachos de basura a lo largo de su extensión, ubicados al inicio, centro y final del puente; pero estos no cuentan con clasificación de desechos. No cuenta con rampas de accesibilidad para personas con discapacitadas, y la seguridad es brindada por una empresa privada, pero se observa que a lo largo del puente sólo se encuentran dos guardias, ubicados al inicio y final del puente. Tiene iluminación a lo largo de su extensión y posee iluminaria en los contornos del puente reflejando al Estero Salado, aunque, por otra parte, la falta de iluminación del manglar que es cercano al Malecón de la Universidad de Guayaquil, hace que la vista nocturna no sea atractiva. 


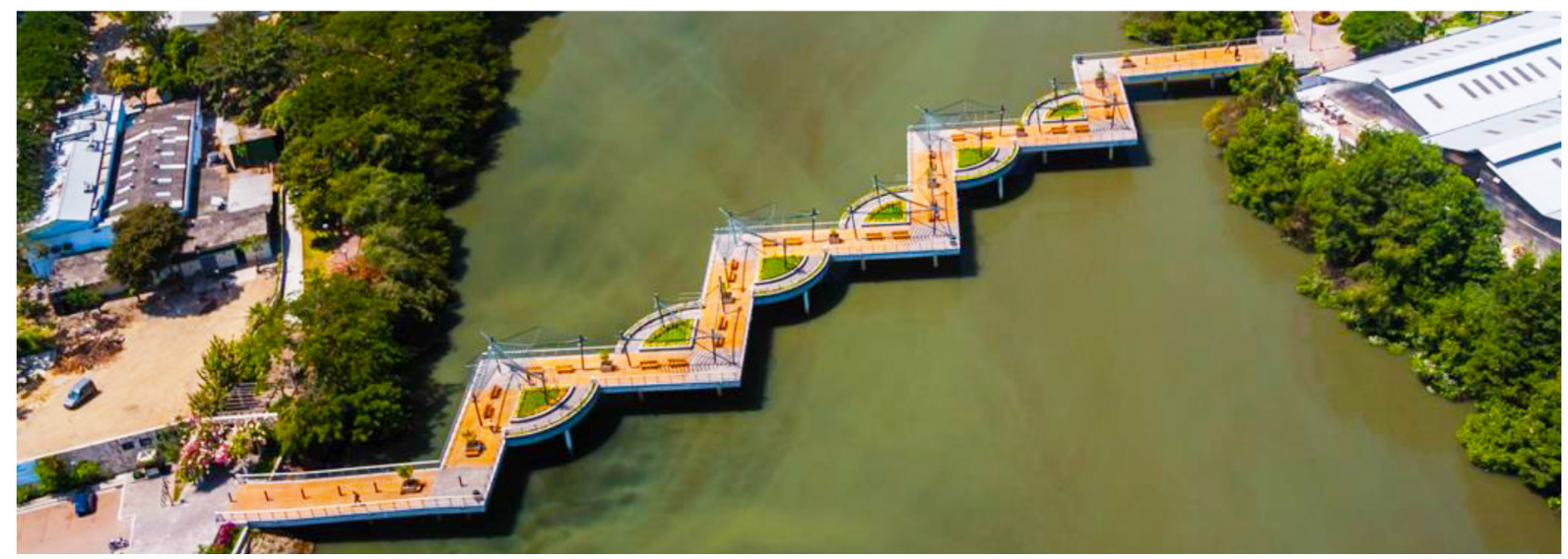

Figura 1. Paseo de la Juventud en la ciudad de Guayaquil, Ecuador.

El Paseo de la Juventud cuenta con dos atractivos turísticos que entran en la categorización de sitios naturales, los cuales son el Estero Salado y el Manglar del Salado; sin embargo, esta obra tiene aspectos que aún deben desarrollarse. Se denota la ausencia de turistas nacionales y extranjeros en esta área. Los transeúntes no se detienen a observar los recursos ambientales ni lo consideran como un lugar de recreación, lejos está de ser considerado como zona para el desarrollo cultural o un lugar dentro un circuito turístico, a pesar de ser este puente fruto de la regeneración urbana. La principal limitante es que no cuenta con la implementación de equipamiento que lo califique para estar dentro de los circuitos turísticos de la ciudad de Guayaquil.

Hoy en día el turismo en Ecuador es reconocido como una fuente de ingresos que cual promueve los recursos naturales y las actividades culturales sin perjudicar el medio ambiente. La ciudad de Guayaquil es un lugar turístico que cuenta con grandes atractivos y zonas de recreación las cuales hacen de la visita del turista una experiencia enriquecedora (Castillo, 2016). Según Rivas y Magadán (2012), un plan de dinamización de un producto turístico "es una herramienta de cofinanciación de las estrategias turísticas", las cuales son desarrolladas en cooperación con las distintas administraciones públicas con la finalidad de colaborar con las entidades locales y mejorar las ofertas turísticas. Esto significa que los planes de dinamización turística son instrumentos de intervención realizados para conseguir una mejora de calidad de destinos turísticos. Según Brunet et al., 2003 "la nueva generación de planes significa figuras de interés en la consolidación de un modelo turístico competitivo y sostenible”. Román (2011) señala que los principales objetivos de los planes de dinamización turística son "la puesta en valor y el uso de recursos turísticos, el estudio y diseño de políticas y estrategias de producto, precio, promoción y comercialización, el desarrollo de una oferta de servicios turísticos de calidad y la creación de nuevos productos turísticos establecido en la explotación creativa de recursos siendo respetuosos con el medio ambiente".

Según Boullón (2016), el espacio turístico es la consecuencia de la presencia y distribución territorial de los atractivos turísticos. Este elemento del patrimonio turístico, incluidos la planta y la infraestructura turística es suficiente para definir el espacio turístico en cualquier país. La mejor forma de determinar un espacio turístico es observar la distribución territorial de los atractivos a fin de detectar las agrupaciones y concentraciones. Esta teoría es la principal causa para organizar todo lo que se va a ejecutar en la zona que se requiere sea un aporte a la oferta turística. Por lo tanto, es necesario llevar a cabo un análisis de todos los elementos del espacio turístico para luego hacer una proyección de la demanda. Schroeder (2011), con la perspectiva de Hiernaux (1989), sugiere que las actividades recreativas en los espacios turísticos "tienden a una apropiación privada de ciertas porciones 'atractivas' del territorio (...) a las que ciertos grupos sociales tendrán acceso restringido, haciendo un uso intensivo del mis- 
mo". Villafuerte (2012) expresa que los espacios urbanos constituyen escenarios de ocio y turismo asociados a la cultura, permitiendo colocar estrategias sobre el modelo y la renovación de la imagen del espacio. El Paseo de la Juventud es un espacio físico ideal para dar acceso a espacios artísticos y culturales, debido a que no existe una extensión más amplia para poder implementar otro tipo de actividad que lo convierta en un aporte a la oferta turística.

Por otra parte, Vázquez (1998) explica que el desarrollo local es un proceso de crecimiento económico que tiene como objetivo una mejora en el nivel de vida de los habitantes, teniendo en cuanta las dimensiones económica, sociocultural, política y ambiental. Barredo (2010) explicita que el objetivo del desarrollo territorial es establecer un modelo de desarrollo cooperativo, sustentable y equilibrado con un profundo sentido de identidad. Asimismo, Panosso y Guilherdem (2012) exponen el modelo de Leiper (1990) de la siguiente manera: Leiper (1990) propuso un modelo de sistema turístico compuesto de cinco elementos que comprenden tres elementos geográficos: la región de origen del viajante, una región de tránsitos que intercomunica el origen con el destino y la región del destino turístico. Los otros dos elementos son el turista y la industria de turismo y viajes. La interacción de estos cinco elementos es influida por factores externos y, a su vez, este mismo sistema impacta sobre los diferentes ambientes como el humano, el sociocultural, el económico, tecnológico, físico, político, legal, etc., e influye a medida que los viajantes pasan a través de la región de tránsito.

Respecto del desarrollo sustentable, Fernández y Gutiérrez (2008) proponen tres ejes analíticos. Primero, un desarrollo que tome en cuenta la satisfacción de las necesidades; segundo, que respete al medio ambiente; y tercero, que no sacrifique los derechos de las generaciones futuras. Un desarrollo sostenible es una de las propuestas para lograr un avance turístico de forma que se ayude a conservar el medio ambiente.

Tirado (2013) define la Teoría de Deming, aplicada a un plan de dinamización de un producto turístico, como "una metodología aconsejada para la realización de cualquier actividad que permite lograr unos resultados, partiendo de información fiable para la toma de decisiones”. El ciclo de Deming consiste en cuatro partes: planear, hacer, verificar, actuar. La importancia de realizar el ciclo de Deming en un plan de dinamización es que las cuatro fases se basan en que los objetivos se realicen de manera adecuada y si se comete algún error definiendo las metas o en alguno de los pasos se puede volver a replantear todo, haciendo un mejoramiento continuo.

En este trabajo el objetivo fue analizar cómo influye la concurrencia de turistas en el Paseo de la Juventud, mediante un estudio de campo para proponer un plan de dinamización que permita su inserción en la oferta dentro del circuito turístico de Guayaquil.

\section{METODOLOGÍA}

\section{Marco conceptual}

En este trabajo se usó como marco conceptual la Teoría del Espacio Turístico y la Teoría de Deming aplicada a un plan de dinamización de un producto turístico. Este es un estudio descriptivo, con un enfoque cualitativo y cuantitativo. El método cualitativo se usó para presentar resultados con base a toda la información recopilada de la población y de las entrevistas. El enfoque cuantitativo permitió la recolección de datos estadísticos para el estudio y análisis de resultados de las encuestas.

Una de las técnicas usadas para recopilar datos fue la de la observación, la que permite al investigador tener una información más oportuna y real de lo que sucede. Se realizaron encuestas a los habitantes de la ciudad de Guayaquil para conocer sus expectativas sobre el Paseo de la Juventud, con preguntas cerradas y objetivas. También se realizaron entrevistas a operadores turísticos y al Gerente de la Fundación Siglo XXI; ambas entrevistas fueron de formato diferente porque los puntos de vista y las personas a entrevistar eran diferentes. Se usaron diversos instrumentos de recopilación como cámara fotográfica, computadoras, celulares, cuaderno de apuntes, pluma, etc. Para la elaboración de esta propuesta se realizó un análisis de situación actual del Paseo de la Juventud, así como del entorno.

\section{Tamaño de la muestra}

La muestra es la parte de la población que se selecciona, de la cual realmente la información para el desarrollo del estudio y sobre la cual se efectuarán la medición y la observación de las variables objeto de estudio (Bernal, 2006). Una vez definida la población que transita semanalmente en el Paseo de la Juventud (27 150 personas) se determinó 
el tamaño de la muestra (n) considerando un $95 \%$ de confianza, error del $5 \%$ y varianza máxima. Se obtuvo $n=379$.

\section{RESULTADOS}

La muestra considerada en este estudio consideró 146 hombres (39\%) y 233 mujeres (61\%). Del total de encuestados se pudo observar que el 69\% (263 personas) se encuentra dentro del rango de edad entre 18 y 25 años, el $22 \%$ (83 personas) se encuentra dentro del rango de edad entre 25 y 35 años. Este resultado es consistente con el hecho que las personas que transitan por el Paseo de la Juventud en su mayoría son estudiantes de la Universidad Católica Santiago de Guayaquil y la Universidad de Guayaquil. El 98\% de los encuestados (370 personas) son ecuatorianos, mientras que el $2 \%$ (sólo 9 personas) son extranjeros provenientes de distintos países (México, Perú, Colombia, España, Estados Unidos e Italia). Se puede observar que el Paseo de la Juventud es conocido por el $78 \%$ de los encuestados (269 personas), mientras que el $22 \%$ (100 personas) no conoce este puente. Esta información es relevante para mantener el interés dentro de las personas que lo frecuentan y para dar a conocer el puente a futuros turistas.

Con respecto a la frecuencia de visita, el $39 \%$ de los encuestados (119 personas) frecuentan el Pase de la Juventud lugar de lunes a viernes, tomando en consideración que son estudiantes los que lo transitan. Por otra parte, el $30 \%$ (90 personas) lo frecuentan una vez a la semana, el $29 \%$ ( 86 personas) una vez al mes y sólo el $2 \%$ (7 personas) los fines de semana. Tomando en cuenta que la encuesta fue realizada de lunes a viernes en la tarde y el fin de semana por la mañana, se puede observar que el puente es subutilizado como paso de tránsito.

Dentro de los factores planteados que deben ser mejorados en el Paseo de la Juventud están en primer lugar los olores con el $31 \%$, la seguridad con el $27 \%$, el espacio de recreación con el $18 \%$, la iluminación con el $14 \%$, la existencia de tachos de basura con clasificación de residuos con el $7 \%$ y la señalética con el 3\%. Esta información es importante ya que permite plantear estrategias para mejorar los aspectos en base a las expectativas de quienes frecuentan el Paseo de la Juventud.

Las implementaciones que se plantean como más nece- sarias en el Paseo de la Juventud están los eventos culturales con el $28 \%$, fuentes de agua con el $26 \%$, que el puente se convierta en un punto de información turística de la ciudad con el $21 \%$, los jardines florales con el $11 \%$, cerramiento de cristal climatizado con 10\%, y binoculares para aviturismo con el $4 \%$. De la misma manera, los encuestados realizaron recomendaciones respecto a la instalación de cámaras de seguridad, baños públicos, áreas cubiertas, shows para niños y actividades familiares los fines de semana, además de puntos de información para el cuidado del Manglar del Estero.

Dentro de los productos que se prefiere que se vendan dentro del Paseo de la Juventud se sugirió abastecimiento de bebidas (agua, colas, jugos) con el $35 \%$, snacks salados con el $25 \%$, snacks dulces con el $21 \%$, y helados con el $19 \%$.

Del total de encuestados el 95\% (358 personas) asistirían a un evento cultural que se realice en el Paseo de la Juventud, mientas que el $5 \%$ ( 21 personas) no asistirían. Este resultado indica que las personas tienen interés en eventos ya sean de recreación o culturales al aire libre. El 92\% (350 personas) consideran que el Paseo de la Juventud podría considerarse como un punto turístico de la ciudad de Guayaquil, mientras que el $8 \%$ ( 29 personas) no lo consideran. De acuerdo a lo planteado las personas considerarían el Paseo de la Juventud como punto turístico, una vez que se realicen los cambios respectivos para que se cumplan sus expectativas.

Se puede observar que dentro de los medios por los cuales los turistas se informan sobre la promoción de sitios turísticos el preferido son las redes sociales con el $51 \%$, la televisión con el $28 \%$, la radio con el $12 \%$ y las páginas web oficiales de los sitios con el $9 \%$.

Las entrevistas realizadas a un Agente de Viajes y un Concejal del Municipio fueron fundamentales para realizar la propuesta del plan de dinamización. Ambos coincidieron que a pesar que no se realicen tantas actividades esperadas, el Paseo de la Juventud además de ser un puente solo de paso, tiene un gran potencial para ser considerado un punto turístico de Guayaquil, por su cercanía con emblemáticas universidades, cerca de dos calles principales y del parque lineal y Malecón del Salado. El proyecto le pareció interesante al Concejal del Municipio, el cual especificó que podría ser financiado por Fundación Siglo XXI para 
que la ciudad de Guayaquil pueda tener otro atractivo innovador. Por este motivo se considera un proyecto viable. El plan de dinamización tiene como objetivo general insertar el Paseo de la Juventud dentro de la oferta turística de la ciudad de Guayaquil a través de la implementación de estrategias y acciones para la mejora de su gestión y promoción. El plan considera las siguientes líneas estratégicas (LE) / acciones (A):

- $\quad$ LE1 Promoción turística

- A1.1 Creación de redes sociales y página web

- A1.2 Posicionamiento en la oferta turística LE2 Mejoras del Paseo de la Juventud

- A2.1 Equipamiento

- A2.2 lluminación y seguridad

- A2.3 Aspecto ambiental

\section{LE1 Promoción turística / A1.1 Creación de redes sociales y página web}

Los objetivos son ofrecer información actualizada de las gestiones que se realicen en el Paseo de la Juventud; informar a los visitantes de actividades culturales y de recreación mediante el desarrollo de un calendario, además de atractivos cercanos y lugares que se pueden visitar en Guayaquil; y dar a conocer a los turistas información sobre los atractivos turísticos cercanos y cómo cuidarlos. Las acciones son la selección de información que se va a incluir en la página web, con un diseño llamativo y dinámico, que sea de fácil manejo para quien visite la página; activación de un correo electrónico creado para diferentes aspectos (espacio para registro por medio de correo electrónico para recibir información sobre las actividades que se realicen, consultas y sugerencias); opción de registro para solicitar espacio para realizar actividades (ferias para emprendedores, exposiciones de música y arte); concientización a los visitantes, transeúntes diarios y turistas sobre los atractivos que tiene a su alrededor el Paseo de la Juventud y una guía de cómo cuidarlos; e interacción en las cuentas oficiales de Facebook, twitter e Instagram del Paseo de la Juventud para que los visitantes sepan de las mejoras y eventos dentro del mismo.

\section{LE1 Promoción turística / A1.2 Posicionamiento en la oferta turística}

Los objetivos son dar a conocer al Paseo de la Juventud entre los viajeros, las agencias de viajes y tour operadores; y establecer al Paseo de la Juventud como punto de información turística de Guayaquil. La acciones son proponer a la Empresa Pública Municipal de Turismo y Promoción Cívica la inserción del Paseo de la Juventud a la oferta turística de Guayaquil para que se convierta en un lugar de recreación visitado por nacionales y extranjeros; colocar información en páginas web de la ciudad sobre el Paseo de la Juventud y sus gestiones; y realizar convenios con empresas de turismo para que promocionen el Paseo de la Juventud a cambio de que en los puntos de información turística del puente se promocione a las mismas.

\section{LE2 Mejoras del Paseo de la Juventud / A2.1 Equipamiento}

Los objetivos son mejorar los espacios del Paseo de la Juventud para que sea más llamativo para los turistas; y lograr que el Paseo de la Juventud sea un área recreativa más no un área de tránsito. Las acciones son la construcción de fuentes de agua ubicadas en cuatro jardineras del puente; implementación de máquinas dispensadoras de bebidas y snacks para los turistas; instalación de dos pantallas touch donde tendrán información del Paseo de la Juventud, de los atractivos cercanos y de Guayaquil en general; e instalación de dos binoculares para facilitar e impulsar el aviturismo, ubicados en las dos puntas céntricas del Paseo de la Juventud (estos binoculares facilitarán además la observación del manglar).

\section{LE2 Mejoras del Paseo de la Juventud / A2.2 lluminación y seguridad}

El objetivo es brindar seguridad y comodidad a los transeúntes frecuentes y turistas del Paseo de la Juventud. Las acciones son la instalación de cámaras de seguridad a través del sistema Ojos de Águila en las entradas y a la mitad para hacer del Paseo de la Juventud un lugar más seguro; e instalación de iluminaria para las fuentes de agua para que la visita del Paseo sea más llamativa.

\section{LE2 Mejoras del Paseo de la Juventud / A2.3 Aspecto ambiental}

Los objetivos son cuidar del estero y manglar, los cuales son los atractivos que posee el Paseo de la Juventud; y promover el cuidado del medio ambiente, haciendo énfasis en los atractivos del Paseo de la Juventud. Las acciones incluyen el cambio de tachos de basura normales por tachos con clasificación de residuos, los cuales estarán ubicados al inicio y al final del Paseo de la Juventud; y la instalación de fuentes de agua con filtros necesarios para procesar el agua del estero y ayudar a la oxigenación del mismo. 


\section{CONCLUSIONES}

A partir del estudio de la situación actual y modificada, y los resultados obtenidos, se pueden plantear las siguientes conclusiones:

- La ciudad de Guayaquil tiene una gran variedad de atractivos que la embellecen y hacen del turismo una de las actividades económicas importantes para la misma. La Municipalidad de Guayaquil ha desarrollado una serie de regeneraciones urbanas que le ha permitido a la ciudad tener un desarrollo. Entre las obras de la regeneración urbana se encuentra el Paseo de la Juventud, el cual adoptó su nombre después de su re-inauguración; la finalidad de este cambio era promover el arte y la cultura entre los jóvenes guayaquileños, aunque no se realizaron gestiones u organización de actividades que respalden dicho cambio.

- Después del análisis de la situación actual del Paseo de la Juventud se pudo determinar mediante observación directa que las personas utilizan el puente como paso de tránsito y no como área de recreación y que en su mayoría son jóvenes pertenecientes a las universidades cercanas. Además, se determinaron una serie de aspectos que deberían ser cambiados o mejorados, los cuales fueron también tomados como resultado de las encuestas realizadas para recolectar información.

- A través de encuestas se establecieron implementaciones o mejoras que serían deseables desde el punto de vistas del público, para que sean realizadas en pro del desarrollo de una imagen turística que permita al Paseo de la Juventud ser insertado en los circuitos turísticos de Guayaquil.

- La propuesta planteada en el plan de dinamización tiene dos ejes principales: La implementación o mejoras desde la infraestructura, iluminación y seguridad hasta un aspecto que se enfoque al ámbito ambiental y, por otra parte, la promoción turística y su inserción en el medio turístico.

- Después de esta investigación se recomienda el análisis respectivo para la factibilidad de la implementación de un plan de dinamización, el cual una vez ejecutado permitirá al Paseo de la Juventud contar con elementos necesarios que se ajuste a las expectativas de los turistas y a su vez a los requerimientos para la inserción al circuito turístico de la ciudad de Guayaquil. La inserción del Paseo de la Juventud a Rutas de la ciudad de Guayaquil, tanto la Ruta del Aviturismo de la Municipalidad como la Ruta de la Orilla del Guayas que pertenece a las rutas opcionales creadas por el Diario El Universo.

- Considerando que uno de los puntos del plan de dinamización está ligado a la parte ambiental, se recomienda seguir con los proyectos de limpieza, oxigenación y restauración del Estero Salado que lleva a cabo del Ministerio del Medio Ambiente, para continuar con el progreso de uno de los aspectos relevantes como los olores del Estero.

\section{REFERENCIAS}

André, M., Cortés, I. y López, J. (s.f.). Turismo Cultural: cuando el recurso cultural supera al destino turístico. El Caso de Figueres. España: Universidad de Barcelona.

Banco Central del Ecuador. (s.f.). Indicadores Económicos. Recuperado el 20 de Junio del 2016, de bce.fin.ec: https://www.bce.fin.ec/index.php/component/k2/ item/754

Banco Mundial. (2016). Datos estadísticos de Ecuador. Recuperado el 20 de Junio del 2016, de datos.bancomundial.org: http://datos.bancomundial.org/ pais/ecuador

Barredo, G. (1998). Aportación al inicio de una experiencia de desarrollo socio-económico; basada en los principios cooperativos, en una comunidad indígena del estado de Yucatán. El caso de Telchach pueblo. [Lectura en PDF]. Recuperado de: http://www. eumed.net/tesis-doctorales/2014/gb

BBC. (2015). La millonaria apuesta de Ecuador en el Super Bowl. Recuperado el 25 de Junio del 2016, BBC.com: http://www.bbc.com/mundo/noticias/2015/02/150130_economia_ecuador anuncio_superbowl

Bernal, C (2006). Metodología de la Investigación: Administración, Economía, Humanidades y Ciencias Sociales. Colombia: Pearson Education.

Boullón, R. (2006). Planificación del Espacio Turístico. México: Trillas. 
Brunet, P., Almeida, F., Coll, M. y Monteserín, O. (2005). Los Planes de Excelencia y Dinamización Turística (PEDT), un instrumento de cooperación a favor del desarrollo turístico. España.

Calero, R. (2010). Gobernanza del Estero Salado.

Castillo, M. (2016). Estudio de las facilidades turísticas orientado a niños y adolescentes para el turismo de aventura en la ciudad de Guayaquil, Provincia del Guayas.

Diario El Expreso. (2015). Guayaquil crea un observatorio turístico. Recuperado el 26 de Junio del 2016, de Expreso.ec: http://expreso.ec/actualidad/ guayaquil-crea-un-observatorio-turistico-KAGR_7679750

Diario El Expreso. (2016). Los turistas redefinen la ruta de sus visitas. Recuperado el 26 de Junio del 2016, Expreso.ec: http://expreso.ec/guayaquil/los-turistas-redefinen-la-ruta-de-sus-visitas-DN438445

Diario EI Universo. (2012). Puente Zigzag ya está listo. Recuperado el 9 de Junio del 2016, de El Universo.com: http://www.eluniverso. com/2012/06/06/1/1445/puente-zigzag-ya-esta-listo.html

Diario El Universo. (2015). Ruta de la orilla del Guayas. Recuperado el 11 de Junio del 2016, de El Universo.com: http://www.eluniverso.com/noticias/2015/07/20/nota/5029816/ruta-orilla-guayas-estero-salado

Fernández, L. y Gutiérrez, M. (2013). Bienestar Social, Económico y Ambiental para las presentes y futuras generaciones. México: Universidad Autónoma Metropolitana unidad Azcapotzalco.

García, A., Ramírez, A. y Sánchez, J. (2004). El Desarrollo Sustentable: Interpretación y Análisis. Revista del Centro de Investigación. Universidad La Salle, 60 55-59.

Guayaquil es mi Destino. (s.f.). Ruta del Malecón del Estero Salado y el Parque Lineal. Recuperado de: http://www.guayaquilesmidestino.com/es/rutas/ aviturismo-urbano/primera-ruta-urbana-aviturismo-guayaquil-malecon-del-estero-salado-y-parque-lineal

Guayaquil es mi Destino (s.f.). Observatorio turístico de Guayaquil. Recuperado el 20 de Julio del 2016, de guayaquilesmidestino.com: http://www.guayaqui- lesmidestino.com/sites/default/files/observatorio-turistico-guayaquil-es-mi-destino.pdf

Guayaquil Siglo XXI. (s.f.). Reglamento de "Guayaquil Siglo XXI, Fundación Municipal para la Regeneración Urbana”. [Lectura PDF] Recuperado de https:// www.guayaquilsigloxxi.info/la-fundacion/

INEC. (2010). Resultado del Censo del 2010 de población y vivienda. Recuperado de: http://www.ecuadorencifras.gob.ec/wp-content/descargas/Manu-lateral/Resultados-provinciales/guayas.pdf

INEC. (s.f.). Así es Guayaquil cifra a cifra. Recuperado de: http://www.ecuadorencifras.gob.ec/documentos/web-inec/Infografias/asi_esGuayaquil_cifra_a_cifra.pdf

Jaramillo, S. (2014). Análisis del turismo urbano como modalidad de desarrollo turístico para la ciudad de Guayaquil.

Ministerio del Medio Ambiente. (s.f.). Plan de superoxigenación del Estero Salado de Guayaquil. Recuperado de: http://www.ambiente.gob.ec/plan-de-superoxigenacion-del-estero-salado-de-guayaquil/

Ministerio del Ambiente (2014). Ley de gestión ambiental. Recuperado de: http://www.ambiente.gob. ec/wp-content/uploads/downloads/2012/09/ LEY-DE-GESTION-AMBIENTAL.pdf

Ministerio del Ambiente. (s.f.). Descontaminación del Estero Salado avanza en diferentes frentes de intervención. Recuperado de: http://www.ambiente.gob.ec/descontaminacion-del-estero-salado-avanza-en-diferentes-frentes-de-intervencion/

Ministerio de Turismo. (2007). Plan Estratégico de Desarrollo de Turismo Sostenible para Ecuador "PLANDETUR 2020". [Lectura en PDF] Recuperado de: http://www.turismo.gob.ec/wp-content/uploads/ downloads/2013/02/PLANDETUR-2020.pdf

Ministerio de Turismo. (2015). Ingreso de divisas por turismo creció en un $21 \%$ hasta septiembre del 2014. Recuperado de: http://www.turismo.gob. ec/ingreso-de-divisas-por-turismo-crecio-en-un21-hasta-septiembre-del-2014/

Monserrate, B. y Medina, J. (2011). Estudio de condiciones físicas, químicas y biológicas en la zona intermareal de dos sectores del Estero Salado con diferente desarrollo urbano. 
Moreno, M. (s.f.). La tierra húmeda Humedales ecuatorianos. [Artículo Web] Recuperado de: http://www. paramo.org/

Municipalidad de Guayaquil. (2014). La Municipalidad. [Página Web] Recuperado de: http://www.guayaquil.gov.ec/la_municipalidad

Organización Mundial del Turismo. (s.f.). En Cuadernos de Turismo. Recuperado de: http://vidasilvestre.org. uy/wpcontent/uploads/2012/07/Turismo-y-Sustentabilidad_de-la-Teor\%C3\%ADaa-la-Pr\%C3\%A1ctica-en-Cubā Cuadernos-de-Turismo.pdf

Panosso, A. y Lohmann, G. (2012). Teoría del Turismo: conceptos, modelos y sistemas. México: Trillas.

Román, A. (2011). Planes de Dinamización y Excelencia
Turística versus Municipios Turísticos: problemática, similitudes y diferencias esenciales. España: Universidad de Granada.

Schroeder, R. (2011). La actividad turístico-recreativa en la reestructuración del espacio urbano. El caso de una ciudad media Bahía Blanca, Argentina. [Artículo Web] Recuperado de: http://revistas. uexternado.edu.co/index.php/tursoc/article/ view/3125/3507

Secretaría Nacional de Planificación y Desarrollo. (2010). Plan Nacional del Buen Vivir. [Lectura en PDF] Recuperado de: http://www.buenvivir.gob. ec/versiones-plan-nacional http://www.buenvivir. gob.ec/versiones-plan-nacional 